Pacific Journal of Mathematics

A NOTE ON QUADRATIC FORMS OVER PYTHAGOREAN 


\title{
A NOTE ON QUADRATIC FORMS OVER PYTHAGOREAN FIELDS
}

\author{
ROGER WARE
}

\begin{abstract}
A theorem of T. A. Springer states that if $F$ is a field of characteristic not two and $L$ is an extension field of $F$ of odd degree then any anisotropic quadratic form over $F$ remains anisotropic over $L$. A weaker version (and an immediate consequence) of this theorem says that the natural map $r: W(F) \rightarrow W(L)$, from the Witt ring of $F$ to the Witt ring of $L$, is injective. This note investigates the relationship between these statements in the case that $L$ is a finite Galois extension of a pythagorean field $F$. Specifically, it is shown that if $r$ is injective then any anisotropic quadratic form over $F$ remains anisotropic over $L$ and if, in addition, $L$ is pythagorean then the extension must be of odd degree. An example is provided of a Galois extension of even degree with $r$ injective.
\end{abstract}

Notations and terminology in this paper will follow [4]. Thus by a field $F$ we shall mean one of characteristic different from two and $W(F)$ will denote the Witt ring of anisotropic quadratic forms over $F$. If $F \subset L$ is an extension of field then $r_{L / F}: W(F) \rightarrow W(F)$ will denote the induced homomorphism of Witt rings. When there is no possibility of confusion we shall simply write $r$ in place of $r_{L / F}$. In general, the mapping $r$ will fail to be injective. However, if $F \subset L$ is an extension of odd degree then the above mentioned theorem of Springer will imply the injectivity of $r[4$, Chapter $7, \S 2]$. In the case of ordered (= formally real) fields, information about the kernel of $r$ can be used to yield information about extending orderings. Specifically, every ordering on $F$ extends to an ordering on $L$ if and only if Ker $r$ is a nil ideal of $W(F)$ [3, Corollary 2.11]. One can use this, together with Springer's theorem, to recover the fact that if $F \subset L$ is an extension of odd degree with $F$ formally real then every ordering on $F$ extends to $L$. Moreover, if $F$ is pythagorean then $W(F)$ has no nonzero nilpotent elements [4, Theorems 3.3 and 6.1, pp. 236 and 248] so for any extension $L$ of $F, r: W(F) \rightarrow W(F)$ is injective if and only if every ordering on $F$ extends to $L$.

Proposition 1. Let $F \subset L$ be a finite Galois extension of degree $n$ with $L$ pythagorean. If $r: W(F) \rightarrow W(L)$ is injective then $n$ is odd.

Proof. Let $G$ be the Galois group of the extension $F \subset L$, let $H$ be 
a 2-Sylow subgroup of $G$, and let $K=L^{H}$ be the fixed field of $H$. Then $K$ is also pythagorean [4, Exercise 17, p. 254].

If $F$ is not formally real then every element of $K$ is a square in $K$ (i.e. $K$ is "quadratically closed"). Thus, from Galois theory, $H$ must be trivial and hence $G$ is a group of odd order.

Now assume $F$ is formally real and let $<$ be an ordering on $F$. Since $r: W(F) \rightarrow W(L)$ is injective, < extends to $L$ (and to $K)$. Moreover by [2, Exercise 2, p. 289], < extends to exactly [ $L: F]$ orderings on $L$ and to $t \leqq[K: F]$ orderings on $K$ (compare [3, Proposition 5.12]). Let $<_{1},<_{2}, \cdots,<_{m}, m \leqq t$, be the orderings on $K$ which extend $<$ and which also extend to $L$. Since $K \subset L$ is a Galois extension, it again follows that each $<_{i}$ extends exactly $[L: K]$ different ways to $L$. Thus $[L: F]=m[L: K]$, which implies that $m=[K: F]$. Hence $m=t$ so that every extension of $<$ to $K$ also extends to $L$. But every ordering on $K$ is the extension of some ordering on $F$, so it follows that every ordering on $K$ extends to $L$. Since $K$ is a pythagorean field, the mapping $r_{L / K}: W(K) \rightarrow W(L)$ is injective. If the Galois group $H$ of the extension $K \subset L$ is not trivial then there will exist a nonsquare $a$ in $K$ with $\sqrt{a}$ in $L$. Then $\langle 1,-a\rangle$ is an anisotropic form over $K$ whose class in $W(K)$ is a nonzero element in the kernel of $r_{L / K}$. Thus $H$ is also trivial in this case, i.e. $n$ is odd.

COROLlaRy. Let $F \subset L$ be a finite Galois extension of degree $n$ with $L$ pythagorean. If every ordering on $F$ extends to $L$ then $n$ is odd.

Proof. By [4, Exercise 17, p. 254], $F$ is also pythagorean.

The following modification of a construction due to Manfred Knebusch shows that the hypothesis that $L$ be pythagorean is essential in Proposition 1 and its corollary.

ExAmpLE. A Galois extension $F \subset L$ of formally real fields with $F$ pythagorean (actually euclidean), $[L: F]$ even, and $r: W(F) \rightarrow W(L)$ injective.

Choose $n \geqq 5$ and let $K$ be a formally real field on which the alternating group $A_{n}$ acts as a group of automorphisms (e.g. $K=$ $\left.\boldsymbol{R}\left(x_{1}, \cdots, x_{n}\right)\right)$. Let $k=K^{A_{n}}$ be the fixed field and let $\tilde{k}$ be the quadratic closure of $k$, i.e. the compositum of all Galois extensions of $k$ with degree a power of $2[4$, p. 219]. Then $\tilde{k}$ is a Galois extension of $k$ and since $[K: k]$ is not a power of two, $K$ is not contained in $k$. Thus $\tilde{k} \cap K \neq K$ is a Galois extension of $k$ so Galois theory and the simplicity of $A_{n}$ imply that $\tilde{k} \cap K=k$.

Now let $R$ be a real closure ([2], [4], [5]) of the formally real field $K$ and let $F=R \cap \tilde{k}$. Then we also have $F \cap K=k$. Moreover, $F$ is formally real and it is easy to see that any $a$ in $F$ is either a square in $F$ 
or the negative of a square in $F$. In particular, $F$ is pythagorean and has exactly one ordering. From Sylvester's law of inertia we have $W(F) \cong Z$ (cf. [4, pp. 42-43]).

Let $L=F K$ be the compositum of $F$ and $K$ in $R$. Then $L$ is a formally real Galois extension of $F$ with Galois group $A_{n}$ [5, Theorem 4, p. 196]. In particular, $[L: F]$ is even. Finally, any signature $\sigma_{<}: W(L) \rightarrow Z$ arising from an ordering $<$ on the formally real field $L$ (see [4, pp. 42-43], [3, p. 211]) will provide a splitting for the map $r: W(F) \rightarrow W(L)$.

Proposition 2. Let $F$ be a pythagorean field and $L$ a finite Galois extension of $F$. Then the following statements are equivalent;

(1) $r: W(F) \rightarrow W(L)$ is injective.

(2) If $q$ is an anisotropic quadratic form over $F$ then $q_{L}=L \otimes_{F} q$ is anisotropic over $L$.

Proof. (1) $\Rightarrow$ (2). If $F$ is not formally real then $F$ is quadratically closed so all anisotrpic forms over $F$ are one dimensional. Hence the implication is obvious in this case.

Now assume $F$ is formally real and let $T r *$ denote Scharlau's transfer map relative to the $F$-linear trace map $\operatorname{Tr}_{L / F}$ (which associates to each quadratic form $q$ over $L$ the $F$-quadratic form $\operatorname{Tr}_{L / F} \circ q$ ) [4, Chapter $7, \S 1,6],[3, \S 5]$. Then for any anisotropic form $q$ over $F$, there is an isometry $L \otimes_{F} \operatorname{Tr}^{*}\left(q_{L}\right) q_{L} \perp \cdots \perp q_{L} \cong[L: F] \cdot q_{L}$, where $[L: F] \cdot q_{L}=q_{L} \perp \cdots \perp q_{L},[L: F]$ times [4, Theorem 6.1, p. 212] compare [3, Corollary 5.10]). Since the mapping $r: W(F) \rightarrow W(L)$ is injective this means that $\operatorname{Tr} *\left(q_{L}\right)$ is isometric to $[L: F] \cdot q$ over $F$. But $F$ is a formally real pythagorean field, so by (the proof of ) [4, Theorem 3.3], $[L: F] \cdot q$ is anisotropic over $F$. Therefore $\operatorname{Tr} *\left(q_{L}\right)$ is anisotropic over $F$ so that, in particular, $q_{L}$ is anisotrpic over $L$.

The implication (2) $\Rightarrow(1)$ is immediate.

It seems to be an open question whether, for an arbitrary extension $F \subset L$, the injectivity of $r: W(F) \rightarrow W(L)$ implies that anisotropic forms over $F$ remain so over $L$. However, for a certain class of pythagorean fields the answer is affirmative. Let $F$ be a formally real field, let $X$ be the set of orderings on $F$, and for $a$ in $F$, let $V(a)=\{<$ in $X \mid a>0\}$. Then the family $V(a)_{a_{\varepsilon} F}$ generates a compact, Hausdorff, totally disconnected topology on $X[3$, Lemma 3.3, Theorem 3.18]. The field $F$ satisfies the Strong Approximation Property (SAP) if given any two disjoint closed subsets $U, V$ of $X$ there is an element $a$ in $F$ which is positive at the orderings in $U$ and negative at the orderings in $V$ (cf. [1, Definition 1.4], [3, Corollary 3.21]).

PRoposition 3. Let $F$ be a formally real pythagorean field satisfying SAP and let $L$ be any extension field of $F$. If $r: W(F) \rightarrow W(L)$ is 
injective then any anisotropic quadratic form over $F$ remains anisotropic over $L$.

Proof. In view of [1, Theorem 5.3 (1)], any anisotropic form $q$ over $F$ can be written $q=\left\langle a_{1}, \cdots, a_{n}\right\rangle$ where either all the $a_{i}$ 's are positive or all the $a_{i}$ 's are negative with respect to some ordering $<$ on $F$. If $r: W(F) \rightarrow W(L)$ is injective then $<$ extends to $L$ so an equation $a_{1} x_{1}^{2}+\cdots+a_{n} x_{n}^{2}=0$ with each $x_{i}$ in $L$ is impossible.

\section{REFERENCES}

1. R. Elman and T. Y. Lam, Quadratic forms over formally real fields and pythagorean fields, Amer. J. Math., 94 (1972), 1155-1194.

2. N. Jacobson, Lectures in Abstract Algebra, vol. III, van Nostrand, Princeton, New Jersey, 1964.

3. M. Knebusch, A. Rosenberg, and R. Ware, Signatures on semilocal rings, J. Algebra, 26 (1973), 208-250.

4. T. Y. Lam, The Algebraic Theory of Quadratic Forms, W. A. Benjamin, Reading, Massachusetts, 1973.

5. S. Lang, Algebra, Addison-Wesley, Reading, Massachusetts, 1965.

Received March 13, 1974. Partially supported by NSF Grant GP-37781.

The University OF Kansas

Current address: The Pennsylvania State University 


\section{PACIFIC JOURNAL OF MATHEMATICS}

\section{EDITORS}

RICHARD ARENS (Managing Editor)

University of California

Los Angeles, California 90024

\section{J. DugundJI}

Department of Mathematics University of Southern California Los Angeles, California 90007

D. Gilbarg and J. Milgram

Stanford University

Stanford, California 94305

\section{ASSOCIATE EDITORS}
E. F. BECKENBACH
B. H. NeumanN
F. WoLF
K. YoshiDA

\section{SUPPORTING INSTITUTIONS}

\author{
UNIVERSITY OF BRITISH COLUMBIA \\ CALIFORNIA INSTITUTE OF TECHNOLOGY \\ UNIVERSITY OF CALIFORNIA \\ MONTANA STATE UNIVERSITY \\ UNIVERSITY OF NEVADA \\ NEW MEXICO STATE UNIVERSITY \\ OREGON STATE UNIVERSITY \\ UNIVERSITY OF OREGON \\ OSAKA UNIVERSITY
}

\author{
UNIVERSITY OF SOUTHERN CALIFORNIA \\ STANFORD UNIVERSITY \\ UNIVERSITY OF TOKYO \\ UNIVERSITY OF UTAH \\ WASHINGTON STATE UNIVERSITY \\ UNIVERSITY OF WASHINGTON \\ AMERICAN MATHEMATICAL SOCIETY
}

The Supporting Institutions listed above contribute to the cost of publication of this Journal, but they are not owners or publishers and have no responsibility for its contents or policies.

Mathematical papers intended for publication in the Pacific Journal of Mathematics should be in typed form or offset-reproduced (not dittoed), double spaced with large margins. Underline Greek letters in red, German in green, and script in blue. The first paragraph or two must be capable of being used separately as a synopsis of the entire paper. Items of the bibliography should not be cited there unless absolutely necessary, in which case they must be identified by author and Journal, rather than by item number. Manuscripts, in duplicate, may be sent to any one of the four editors. Please classify according to the scheme of Math. Reviews, Index to Vol. 39. All other communications should be addressed to the managing editor, or Elaine Barth, University of California, Los Angeles, California, 90024.

100 reprints are provided free for each article, only if page charges have been substantially paid. Additional copies may be obtained at cost in multiples of 50 .

The Pacific Journal of Mathematics is issued monthly as of January 1966. Regular subscription rate: $\$ 72.00$ a year (6 Vols., 12 issues). Special rate: $\$ 36.00$ a year to individual members of supporting institutions.

Subscriptions, orders for back numbers, and changes of address should be sent to Pacific Journal of Mathematics, 103 Highland Boulevard, Berkeley, California, 94708.

PUBLISHED BY PACIFIC JOURNAL OF MATHEMATICS, A NON-PROFIT CORPORATION Printed at Jerusalem Academic Press, POB 2390, Jerusalem, Israel.

$$
\begin{gathered}
\text { Copyright (C) } 1975 \text { Pacific Journal of Mathematics } \\
\text { All Rights Reserved }
\end{gathered}
$$




\section{Pacific Journal of Mathematics}

\section{Vol. 58, No. $2 \quad$ April, 1975}

Zvi Artstein and John Allen Burns, Integration of compact set-valued functions . . . . . . . . . 297

Mark Benard, Characters and Schur indices of the unitary reflection group $[321]^{3} \ldots \ldots \ldots . .309$

Simeon M. Berman, A new characterization of characteristic functions of absolutely continuous

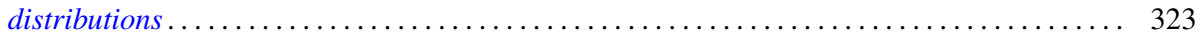

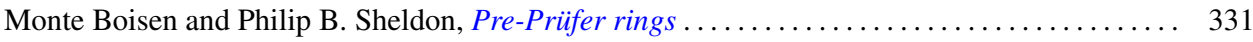

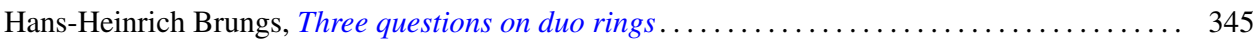

Iracema M. Bund, Birnbaum-Orlicz spaces of functions on groups................. 351

John D. Elwin and Donald R. Short, Branched immersions between 2-manifolds of higher

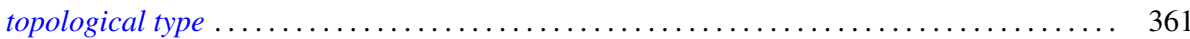

Eric Friedlander, Extension functions for rank 2, torsion free abelian groups . .......... 371

Jon Froemke and Robert Willis Quackenbush, The spectrum of an equational class of

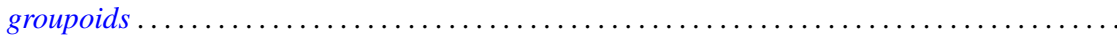

Barry J. Gardner, Radicals of supplementary semilattice sums of associative rings ...........

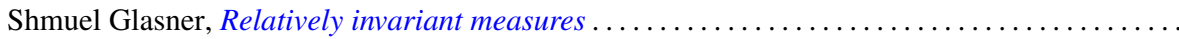

George Rudolph Gordh, Jr. and Sibe Mardesic, Characterizing local connectedness in inverse

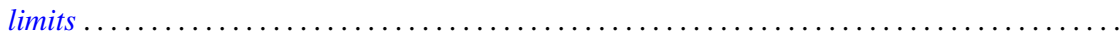

Siegfried Graf, On the existence of strong liftings in second countable topological spaces......

Stanley P. Gudder and D. Strawther, Orthogonally additive and orthogonally increasing

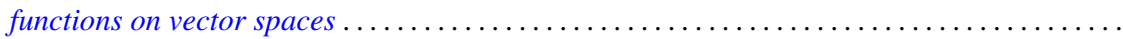

Darald Joe Hartfiel and Carlton James Maxson, A characterization of the maximal monoids and

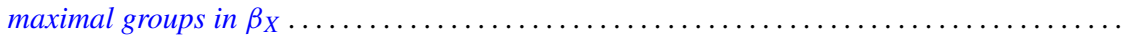

Robert E. Hartwig and S. Brent Morris, The universal flip matrix and the generalized faro-shuffle. .

William Emery Haver, Mappings between ANRs that are fine homotopy equivalences. .

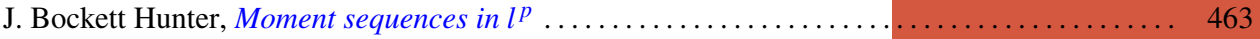

Barbara Jeffcott and William Thomas Spears, Semimodularity in the completion of a poset.... 467

Jerry Alan Johnson, A note on Banach spaces of Lipschitz functions . . . . . . . . . . . . 475

David W. Jonah and Bertram Manuel Schreiber, Transitive affine transformations on

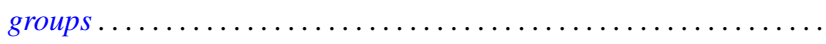

Karsten Juul, Some three-point subset properties connected with Menger's characterization of

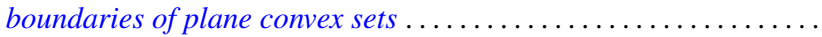

Ronald Brian Kirk, The Haar integral via non-standard analysis . . . . . . . . . . . . . 517

Justin Thomas Lloyd and William Smiley, On the group of permutations with countable

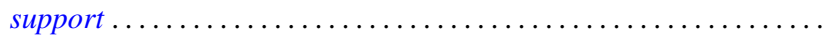

Erwin Lutwak, Dual mixed volumes .................................. 531

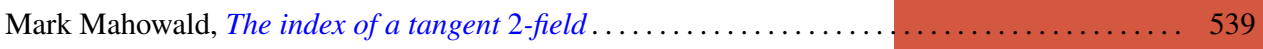

Keith Miller, Logarithmic convexity results for holomorphic semigroups . . . . . . . . . . . . 549

Paul Milnes, Extension of continuous functions on topological semigroups . . . . . . . . . . 553

Kenneth Clayton Pietz, Cauchy transforms and characteristic functions ................ 563

James Ted Rogers Jr., Whitney continua in the hyperspace $C(X) \ldots \ldots \ldots \ldots \ldots \ldots \ldots \ldots .569$

Jean-Marie G. Rolin, The inverse of a continuous additive functional . . . . . . . . . . . . 585

William Henry Ruckle, Absolutely divergent series and isomorphism of subspaces . ........ 605

Rolf Schneider, A measure of convexity for compact sets . ..................... 617

Alan Henry Schoenfeld, Continous measure-preserving maps onto Peano spaces .......... 627

V. Merriline Smith, Strongly superficial elements .......................... 643

Roger P. Ware, A note on quadratic forms over Pythagorean fields . . . . . . . . . . . . . . 651

Roger Allen Wiegand and Sylvia Wiegand, Finitely generated modules over Bezout rings . . . . 655

Martin Ziegler, A counterexample in the theory of definable automorphisms . . . . . . . . . 665 